\section{PROTÓTIPO DE UMA CADEIRA TERAPÊUTICA NEONATAL: DESENVOLVIMENTO, CONFECÇÃO E APLICABILIDADE}

\section{PROTOTYPE OF A NEONATAL THERAPEUTIC CHAIR: DEVELOPMENT, CONFECTION AND APPLICABILITY}

\author{
Graziela Ferreira Biazus ${ }^{1}$, Silvia Raquel Jandt ${ }^{1}$, Cidia Cristina Kupke ${ }^{1}$, \\ Rita C. Silveira ${ }^{2,3}$
}

\section{RESUMO}

Introdução: A longa hospitalização dos neonatos na Unidade de Terapia Intensiva Neonatal tem como consequência o aumento dos riscos de alterações posturais devido ao tempo prolongado dentro de incubadoras e berços. O objetivo do estudo foi desenvolver um dispositivo para promover uma alternativa de mudança de decúbito denominada Cadeira Terapêutico Neonatal.

Métodos: Estudo qualitativo e descritivo de desenvolvimento e produção, aprovado pelo Comitê de Ética em Pesquisa com o n 17-0254 e constituído por três etapas: desenvolvimento e confecção do protótipo; avaliação da aplicabilidade do protótipo por meio de um questionário de aplicabilidade, após transcorridos 10 minutos do posicionamento funcional; e definição do modelo e solicitação do pedido de invenção pelo Instituto Nacional de Propriedade Industrial (INPI).

Resultados: Foram desenvolvidos e confeccionados dois modelos de protótipos de Cadeira Terapêutica Neonatal. Vinte e oito colaboradores da equipe assistencial responderam ao questionário: $82 \%$ consideram muito bom na avaliação no grau de conforto e adaptação do neonato; $96 \%$ avaliaram como muito fácil de realizar o processo de higienização. Onze $(39,28 \%)$ colaboradores sugeriram promover medidas de melhorias no cinto de segurança. Definiu-se o modelo do invento e foi feita a inserção do pedido sob o número BR 20.2020.005865 2 no INPI.

Conclusão: O modelo proposto promoveu uma alternativa de mudança de postura, tendo como característica a aplicabilidade e a usabilidade.

Palavras-chave: Neonato; Posicionamento do paciente; Fisioterapia; Inovação tecnológica; Neuroproteção; Neonatologia

\section{ABSTRACT}

Introduction: The long hospitalization of neonates in the Neonatal Intensive Care Unit has the consequence of increasing the risks of postural changes due to the prolonged time inside incubators and cradles. The aim of the study was to develop a device to promote an alternative for changing the decubitus position called "Neonatal Therapy Chair".

Methods: Qualitative and descriptive study of development and production, approved Human Research Protection Office number 17-0254 - consisting of three stages: Stage 1- development and making of the prototype; Step 2- evaluation of the applicability of the prototype through an Applicability Questionnaire, after 10 minutes of functional positioning; Stage 3- definition of the model and request for an invention request by the NIIP (National Institute of Industrial Property).

Results: Stage 1: two prototype. Stage 2: Twenty-eight employees of the assistance team answered the questionnaire: $82 \%$ consider it very good in assessing the degree of comfort and adaptation of the newborn; $96 \%$ rated it as very easy to carry out the
Clin Biomed Res. 2021;41(3):1-204

1 Serviço de Fisioterapia e Neonatologia, Hospital de Clínicas de Porto Alegre. Porto Alegre, RS, Brasil.

2 Departamento de Pediatria, Universidade Federal do Rio Grande do Sul. Porto Alegre, RS, Brasil.

3 Serviço de Neonatologia, Hospital de Clínicas de Porto Alegre. Porto Alegre, RS, Brasil.

Autor correspondente: Graziela Ferreira Biazus gbiazus@hcpa.edu.br Serviço de Fisioterapia e Neonatologia, Hospital de Clínicas de Porto Alegre

Rua Attílio Bilibio, 251, apto. 1204 E 91530-008, Porto Alegre, RS, Brasil. 
Biazus et al.

cleaning process. Eleven (39.28\%) employees suggested promoting measures to improve seat belts. Step 3: definition of the model of the invention and insertion of the order under number BR 2020200058652 at NIIP.

Conclusion: The proposed model promoted an alternative posture change, having a characteristic of applicability and usability.

Keywords: Newborn; Patient positioning; Physical therapy; Inventions; Neuroprotection; Neonatology

\section{INTRODUÇÃO}

A longa hospitalização dos neonatos na Unidade de Terapia Intensiva Neonatal (UTIN) tem como consequência aumento dos riscos de alterações posturais devido ao tempo prolongado dentro de incubadoras neonatais e berços. As intervenções posturais visam melhorar as aquisições motoras e qualidade de vida ${ }^{1,2}$. O posicionamento do neonato deve ser um cuidado constante, pois envolve toda a dinâmica corpórea, interferindo diretamente sobre as funções fisiológicas primordiais, como a respiração, a função cardíaca e a circulação cerebral ${ }^{3}$. A variação regular da postura e o posicionamento funcional correto enquanto estratégias de cuidado podem atenuar as anormalidades e as assimetrias posturais, favorecendo o desenvolvimento da atividade motora adequada ${ }^{4,5}$.

Estudo desenvolvido por Kochan et al. ${ }^{6}$ na Unidade de Terapia Intensiva Neonatal do Children's Hospital of The King's Daughters in Norfolk, Virginia (USA), apresenta "uma moldura em forma de cunha personalizada" para elevar neonatos dentro das incubadoras modelo Giraffe. Os pesquisadores avaliaram o efeito da elevação e do posicionamento da cabeça da linha média na função cardiopulmonar e na incidência de Hemorragia Peri Intraventricular (HPIV). As principais conclusões foram que a posição elevada a 30 graus e cabeça na linha média nos primeiros 4 dias de vida promove diminuição da probabilidade de HPIV grave e melhora da sobrevida ${ }^{6}$. Em consonância ao tema, a Revisão Sistemática realizada por Romantsik, Calevo e Bruschettini ${ }^{7}$, relata a importância de manter os recém nascidos (RNs) prematuros posicionados com a cabeça na linha média e decúbito elevado nos primeiros dias de vida com objetivo de neuroproteção ${ }^{7}$.

Os RNs prematuros têm um risco aumentado de paralisia cerebral, problemas motores e atrasos cognitivos. Esses frequentemente apresentam deficiências neurocomportamentais (autorregulação, controle do estado, desenvolvimento reflexo, tônus muscular e movimento) que podem ser detectados na idade equivalente a 35-40 semanas de gestação, período da internação na UTIN. Desta forma, recomenda-se promover alinhamento corporal com as mãos próximas da linha média e mudanças posturais para evitar assimetrias ${ }^{8}$.

De acordo com a revisão em Bases de Patentes de criações como Orbit e Patents, INPI (Instituto Nacional de Propriedade Industrial) e Google patents, os pesquisadores não encontraram um invento que apresentasse as características necessárias para promover alternativa de postura dos neonatos dentro de incubadoras e berços. Desta forma, pretendeuse desenvolver um invento denominado "Cadeira Terapêutica Neonatal" para estabelecer uma postura funcional que pudesse ser aplicável na UTIN.

\section{MÉTODOS}

Este estudo foi constituído pela população de neonatos internados em uma UTIN nível II com 32 leitos e em uma UTIN nível III com 20 leitos, em uma área urbana na região Sul do Brasil. Trata-se de um estudo qualitativo e descritivo de desenvolvimento $e$ produção, aprovado pelo Comitê de Ética em Pesquisa, sob o número 170254 , de acordo com a resolução $n^{\circ} 466$ de 2012, com formalização de assinatura do Termo de Compromisso de Utilização de Dados e apoio do Núcleo de Inovação e Transferência de Tecnologia da mesma instituição.

\section{Etapas do projeto}

Etapa 1: Desenvolvimento e confecção do protótipo, elaboração de desenhos, planejamento das dimensões do produto conforme o tamanho das incubadoras neonatais e dos berços, seleção do material a ser utilizado no invento.

Etapa 2: Avaliação da aplicabilidade do protótipo ocorreu por meio do preenchimento de um Questionário. Este questionário foi elaborado pelas pesquisadoras do estudo utilizando como referência Métodos de Pesquisa de Tatiana Engel Gerhardt e Denise Tolfo Silveira ${ }^{9}$, contendo perguntas de múltipla escolha sobre grau de conforto do neonato (ruim, bom, muito bom), segurança (ruim, bom, muito bom) e adaptação do neonato no dispositivo (ruim, bom, muito bom), facilidade no manuseio (muito difícil, fácil, muito fácil) e processo de higienização (muito difícil, fácil, muito fácil) e por fim, uma pergunta aberta, permitindo ao informante responder livremente, usando linguagem própria e emitir opiniões sobre sua observação.

Depois de aceite em participar da pesquisa e assinatura do Termo de Consentimento Livre e Esclarecido (TCLE), os avaliadores formados por profissionais da saúde anônimos alocados na UTIN, preencheram o Questionário de Aplicabilidade após transcorridos 10 minutos do posicionamento funcional do neonato na "Cadeira Terapêutica Neonatal". 
Tempo estimado para ocorrer adaptação do mesmo no dispositivo.

Madlinger-Lewis et al. ${ }^{8}$ desenvolveram um estudo para avaliar os efeitos do posicionamento alternativo em recém-nascidos prematuros em uma UTIN, do qual recomendam a permanência de até duas horas durante três vezes na semana como proposta terapêutica. Dessa forma, foi estipulado como critério de permanência na "Cadeira Terapêutica Neonatal" duas horas.

\section{Critérios de participação dos neonatos no estudo}

Os critérios de inclusão para os neonatos serem posicionados no dispositivo constituíram-se por estar respirando em ar ambiente, sem utilização de tecnologia assistiva como Ventilação Pulmonar Invasiva ou Não Invasiva, TCLE assinado pelos pais e/ou responsáveis, sendo os mesmos informados sobre os objetivos do estudo, assegurando a liberdade de retirar o seu consentimento, se assim o desejassem, em qualquer fase da pesquisa, sem qualquer ônus. Os critérios de exclusão foram: apresentar sinais de desconforto, instabilidade hemodinâmica, fototerapia, ou outro fator durante o tempo em que o neonato estivesse na "Cadeira Terapêutica Neonatal", sendo recomendada sua retirada imediata do estudo.

\section{Intervenções}

A população foi composta pelos recém-nascidos internados em uma UTIN nível II e III. Foram selecionados aleatoriamente para a amostra de acordo com critérios de inclusão $10 \mathrm{RNs}$ prematuros internados na UTIN no período entre Abril a Outubro de 2018 , estes foram posicionados no dispositivo no turno da manhã e/ou tarde, sempre após 30 minutos do término da dieta. Cada RN foi testado uma vez. O invento suporta o peso máximo de 4 quilos. Sendo avaliado o nível de conforto como alteração dos sinais vitais (frequência cardíaca e respiratória, saturação de oxigênio no sangue) e a Neonatal Infant Pain Scale (NIPS) composta por 6 indicadores de dor ${ }^{10}$.

\section{Riscos do estudo}

Caso ocorra a não adaptação do RN no dispositivo como alterações dos sinais vitais e pontuação igual ou maior a 4 na NIPS, este recebe indicação imediata da sua retirada.

Etapa 3: definição do modelo final e solicitação do pedido de invenção pelo INPI (Instituto Nacional de Propriedade Industrial).

O Núcleo de Inovação e Transferência de Tecnologia do Hospital de Clínicas de Porto Alegre e Herval Indústria de Móveis, Colchões e Espumas Ltda colaboraram na execução das Etapas 1 e 3.

\section{RESULTADOS}

Dois protótipos com características semelhantes foram desenvolvidos na Etapa 1: cunha elevada a 45 graus, espuma flexível e tecido impermeável, cinto de segurança com forma triangular (uma ponta inferior fixa e duas pontas laterais móveis, nas regiões laterais dos quadris), com objetivo de estabilizar a região pélvica, prevenindo que o RN desliza-se para baixo, parte interna do dispositivo com desenho anatômico e apoio cervical nas laterais.

Anterior à testagem, o dispositivo recebeu avaliação pelo departamento de Controle de Infecção da Instituição. Este departamento emitiu parecer favorável de utilidade e um guia de orientações para o seu processo de higienização.

Antes de colocar o dispositivo "Cadeira Terapêutica Neonatal" dentro das incubadoras e berços, retira-se o colchão e deixa a plataforma na posição horizontal, não sendo necessárias fixações para estabilizá-lo.

$\mathrm{Na}$ Etapa 2, foram selecionados de acordo com critérios de inclusão dez recém-nascidos prematuros internados na UTIN: com médias de idade gestacional de $30 \pm 1$ semana e de peso ao nascimento de $1458 \pm 102$ gramas. No momento do posicionamento para a avaliação, o tempo médio de internação estava em 2 meses e 15 dias, peso médio de 2426 gramas e idade gestacional corrigida 38 semanas. Nenhum neonato foi excluído do estudo por apresentar alterações clínicas durante o período do posicionamento funcional.

O Questionário de Aplicabilidade foi preenchido por vinte e oito profissionais da saúde anonimamente. As respostas referentes ao grau de conforto, grau de segurança do dispositivo e adaptação do neonato estão presentes na Tabela 1 e avaliação sobre facilidade no manuseio e processo de higienização da "Cadeira Terapêutica Neonatal" apresentados na Tabela 2.

Tabela 1: Resposta de profissionais da saúde anônimos: dados referentes ao grau de conforto, segurança e adaptação do neonato.

\begin{tabular}{lccc}
\hline & $\begin{array}{c}\text { Grau de } \\
\text { Conforto }\end{array}$ & $\begin{array}{c}\text { Grau de } \\
\text { Segurança }\end{array}$ & $\begin{array}{c}\text { Adaptação } \\
\text { do neonato }\end{array}$ \\
\hline Ruim & 0 & $3(10.72 \%)$ & $2(8 \%)$ \\
Bom & $5(18.8 \%)$ & $16(57.14 \%)$ & $3(10.7 \%)$ \\
Muito Bom & $23(82.2 \%)$ & $9(32.14 \%)$ & $23(82.1 \%)$ \\
$\begin{array}{l}\text { Colaboradores } \\
\left(n^{\circ} \text { Total) }\right.\end{array}$ & $28(100 \%)$ & $28(100 \%)$ & $28(100 \%)$ \\
\hline
\end{tabular}


Tabela 2: Resposta de profissionais da saúde anônimos: dados referentes ao manuseio e higienização da "Cadeira Terapêutica Neonatal".

\begin{tabular}{lcc}
\hline & Manuseio & Higienização \\
\hline Muito difícil & $1(3.57 \%)$ & 0 \\
Fácil & $6(21.43 \%)$ & $1(3.57 \%)$ \\
Muito fácil & $21(75 \%)$ & $27(96,43 \%)$ \\
$\begin{array}{l}\text { Colaboradores } \\
\left(\mathrm{n}^{\circ} \text { Total) }\right.\end{array}$ & $28(100 \%)$ & $28(100 \%)$ \\
\hline
\end{tabular}

Em relação à pergunta aberta, onze (39.28\%) profissionais de saúde emitiram opiniões sobre promover medidas de melhorias no cinto de segurança.

$\mathrm{Na}$ etapa 3 foram realizados os ajustes e definição do modelo final do invento: constituindo-se por uma cunha elevada a 45 graus com acomodação anatômica, altura entre $20 \mathrm{~cm}$ e $30 \mathrm{~cm}$, comprimento de $50 \mathrm{~cm}$ a $65 \mathrm{~cm}$ e largura entre 30 e $40 \mathrm{~cm}$, medidas possibilitando inserção dentro das incubadoras e berços neonatais. Composto por espuma flexível de poliuretano de média densidade e revestida por tecido impermeável. A estrutura interna formada por um desenho anatômico, presença de um apoio cervical nas laterais para evitar deslocamento da cabeça e cinto de segurança com quatro pontas para sustentar a região torácica e abdominal (Figuras 1 e 2).

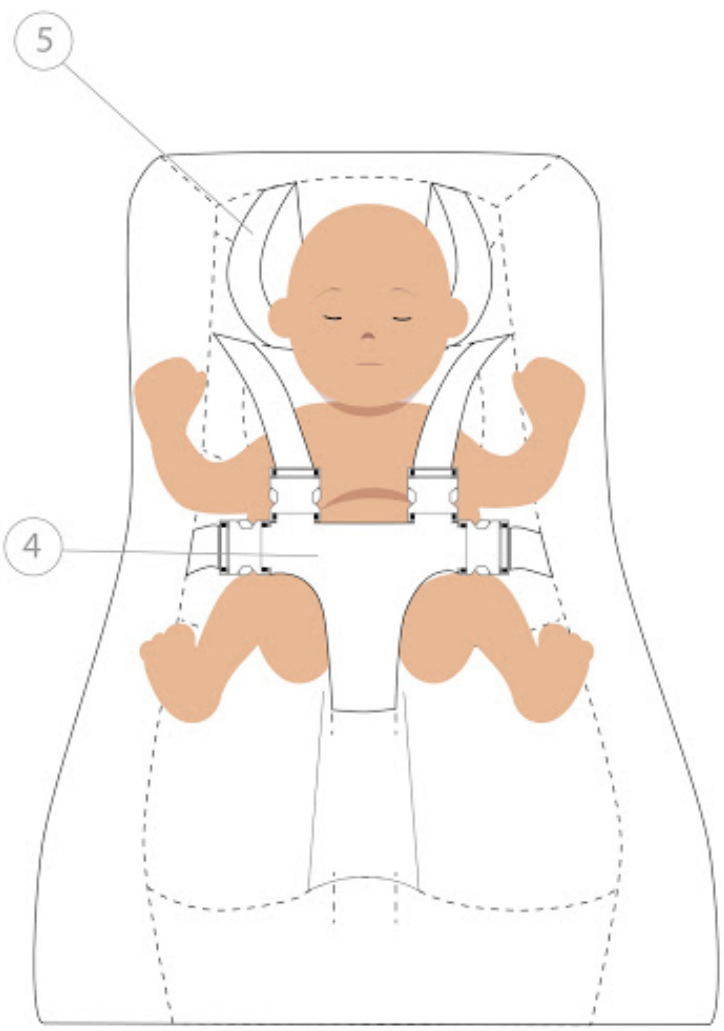

Figura 1: Vista frontal da "Cadeira Terapêutica Neonatal".

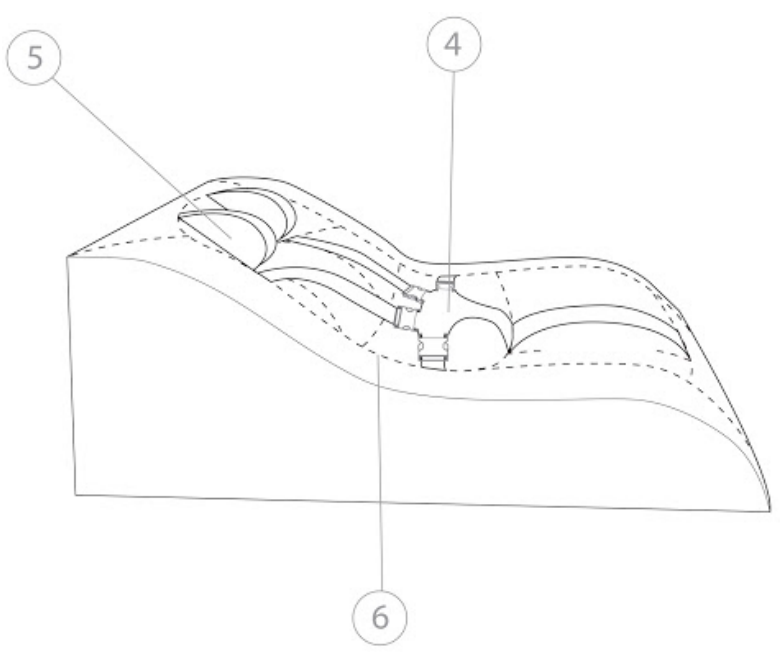

Figura 2: Vista lateral da "Cadeira Terapêutica Neonatal". 4: cinto de segurança com quatro pontas; 5: apoio cervical nas laterais; 6: desenho anatômico.

Após o desenvolvimento do protótipo foi encaminhado o pedido de patente para o INPI (BR 2020200058652 ) em 24/03/2020. O modelo foi registrado na categoria de modelo de utilidade, pois foi baseada no objetivo de uso prático, suscetível a aplicação industrial e tem como resultado uma melhoria funcional no seu uso e na sua fabricação ${ }^{11}$.

\section{DISCUSSÃO}

Foram encontrados na base de pesquisa Bases de Patentes, INPI e Google patents, dois modelos de utilidade que possibilitam posicionamento funcional dos neonatos: sendo o modelo "Dispositivo Construtivo Aplicada em Leitos Envoltório para Bebês e Crianças Pequenas", berço de material acolchoado, sustentado por uma estrutura articulável semelhante a de uma cadeira reclinável, proporcionando um ambiente confortável, reproduzindo uma sensação assemelhada com a do ambiente intra-uterino, tendo como objetivo auxiliar o desenvolvimento físico, neurológico e na prevenção de asfixia em decorrência de refluxo gastroesofágico, indicado para bebês acima de $2000 \mathrm{~g}$. No entanto, tem como principal desvantagem a difícil técnica de higienização e adaptação dentro das incubadoras neonatais, restrita apenas para bebês acima de 2000 g, não aplicável para bebês de baixo peso ao nascimento ${ }^{12}$.

O segundo modelo de utilidade encontrado na literatura foi o dispositivo espanhol "Sistema de Contención y Estimulación Sensorial para Bebés Prematuros", um sistema de contenção de estímulo tátil para neonatos prematuros, possibilitando uma contenção em forma de "ninho", tratando-se de 
um dispositivo robusto, mas não permite elevação a 45 graus da cabeceira ${ }^{13}$.

Na busca pela estabilidade postural ou contenção no ambiente extra-uterino, o neonato fixa-se através de uma hiperextensão cervical, que atuará bloqueando o desenvolvimento da mobilidade e co-contração dessa região. Essa estabilização postural anormal pode acarretar bloqueios sequenciais nas regiões do ombro, pelve e quadris, determinando o atraso no desenvolvimento motor. Um dos primeiros desafios enfrentados por ele é a necessidade de lidar com a organização de sua postura no ambiente extrauterino, sendo incapaz de realizar ajustes posturais devido ao seu baixo tônus muscular e a incapacidade de seus sistemas de organização ${ }^{14}$. Desta forma, o modelo do protótipo "Cadeira Terapêutica Neonatal" difere dos modelos apresentados na literatura e em bases de patentes por apresentar uma alternativa de mudança postural e ajuste postural adequado.

De acordo Alpino et al. o controle postural estável forma a base para organizar e executar o movimento voluntário. É importante para a interação social e a comunicação precoce, pois serve como uma interface com o mundo externo para percepção e ação ${ }^{15}$. Desta forma, foi desenvolvido um invento que facilita as modificações posturais dos neonatos, pretendendo solucionar as dificuldades encontradas de posicionamento no leito. Este modelo visa promover conforto, estabilidade fisiológica e comportamental, objetivando facilitar a biomecânica diafragmática, melhora do sincronismo toracoabdominal, bem como controle postural e alinhamento da cabeça.

Estudos como de Bijl-Marcus et al. ${ }^{16}$, Kochan M et al. ${ }^{6}$ e Bass ${ }^{17}$ relatam a importância do posicionamento do RN prematuro a 30 graus com objetivo de manter adequado fluxo sanguíneo cerebral. Além desse objetivo, a escolha de desenvolver um dispositivo com elevação superior foi possibilitar estimulação neurocomportamental (autorregulação do sistema vestibular e visual, alinhamento da cabeça, postura antigravitária e anti-refluxo). Assim, não recomendamos o posicionamento do neonato em decúbito lateral neste invento.

Estratégias de mudança postural são extremamente importantes para os RNs prematuros, sendo um período crítico para o seu desenvolvimento infantil. A "Cadeira Terapêutica Neonatal" tem como umas de suas indicações na prevenção da plagiocefalia ${ }^{18}$, regulação dos três níveis de controle sensóriomotor dentro do sistema nervoso central ${ }^{19}$ e medida preventiva e tratamento conservador nos casos de refluxo gastroesofágico ${ }^{20}$.

Conclui-se que o invento demonstra propiciar bom grau de conforto e adaptação do neonato, tendo característica de aplicabilidade e usabilidade no ambiente da UTIN. No entanto, futuros estudos serão necessários para avaliar o impacto no tempo de internação hospitalar, função pulmonar e neuroproteção do sistema nervoso central.

\section{Agradecimentos}

Os autores agradecem a Fernanda dos Santos de Oliveira, Núcleo de Inovação e Transferência de Tecnologia do Hospital de Clínicas de Porto Alegre, Enilda Stolzenberg, Fábio Rigon, Felicio Agostini Lampert, Livia Lobel da Luz e equipe assistencial da Unidade de Internação Neonatal do Hospital de Clínicas de Porto Alegre.

\section{Conflitos de Interesse}

Os autores declaram não ter conflitos de interesse.

\section{REFERÊNCIAS}

1. Santos AMG, Viera CS, Toso BRGO, Barreto GMS, Souza SNDH. Aplicação clínica do Procedimento Operacional Padrão de Posicionamento com Prematuros. Rev Bras Enferm. 2018;

71(3):1205-11.

2. Stephens BE, Vohr BR. Neurodevelopmental outcome of the premature infant. Pediatr Clin North Am. 2009;56(3):631-46.

3. Garcia A, Lima M. Estimulação suplementar para o recém-nascido de alto risco. In: Juliani RCTP, Nicolau CM, Paula LCS, Lahóz
ALC, editores. Fisioterapia em UTI pediátrica e neonatal. Barueri: Manole; 2009. p. 133-40.

4. Santos PNL, Dittz ES, Alves CO. Premature newborn babies' positioning at the neonatal intensive care unit and its relation with motor development. Temas Desenvolv. 2011;18(101):22-31.

5. Sociedade de Pediatria de São Paulo, Departamento Científico de Neonatologia. Organização postural do recém-nascido prétermo em unidades neonatais: considerações sobre o uso do ninho [Internet]. São Paulo: Sociedade de Pediatria de São Paulo; 2020 [citado 2021 Mar 10. Disponível em: https://www.spsp.org.br/2020/09/11/ organizacao-postural-do-recemnascido-pre-termo-em-unidadesneonatais-consideracoes-sobre-ouso-do-ninho/.

6. Kochan M, Leonardi B, Firestine A, McPadden J, Cobb D, Shah TA, et al. Elevated midline head positioning of extremely low birth weight infants: effects on cardiopulmonary function and the incidence of periventricularintraventricular hemorrhage. J Perinatol. 2019; 39:54-62.

7. Romantsik O, Calevo MG, Bruschettini M. Head midline position for preventing the occurrence or extension of germinal matrix 
intraventricular hemorrhage in preterm infants. Cochrane Database Syst Rev. 2017;7(7):CD012362.

8. Madlinger-Lewis L, Reynolds L, Zarem C, Crapnell T, Inder T, Pineda R. The effects of alternative positioning on preterm infants in the neonatal intensive care unit: a randomized clinical trial. Res Dev Disabil. 2014;35(2):490-7. Erratum in: Res Dev Disabil. 2015;41-42:101.

9. Gerhardt TE, Silveira DT, editores. Métodos de pesquisa. Porto Alegre: Editora da UFRGS; 2009.

10. Motta GC, Schardosim JM, Cunha ML. Neonatal Infant Pain Scale: CrossCultural Adaptation and Validation in Brazil. J Pain Symptom Manage. 2015;50(3):394-401.

11. Luz LL, Gália CR, Kulzer AM, Sanches PRS. Development, manufacturing and evaluation of effects and safety of a hip orthosis prototype used in the functional rehabilitation of a patient undergoing Girdlestone arthroplasty. Clin Biomed Res. 2018;38(3):301-4.
12. Bueno L, inventor. Dispositivo Construtivo Aplicada em Leitos Envoltório para Bebês e Crianças Pequenas. Patente brasileira MU9001423-5U2. 2012 Dez 26.

13. Diaz ED, González AMC, Colominas MG, Fernandez MG, Urquijo IC, inventores. Sistema de Contención y Estimulación Sensorial para Bebés Prematuros. Patente española ES1180334U. 2017 Abr 7.

14. Barradas J, Fonseca A,

Guimarães CLN, Lima GMS.

A relação entre posicionamento do prematuro no Método Mãe-Canguru e desenvolvimento neuropsicomotor precoce. J Pediatr (Rio J). 2006;82(6):475-80.

15. Alpino C, Compagnone E, Montanaro ML, Cacciatore D, De Luca $A$, Cerulli $A$, et al. Preterm birth and neurodevelopmental outcome: a review. Childs Nerv Syst. 2010;26(9):1139-49.

16. de Bijl-Marcus K, Brouwer AJ, De Vries LS, Groenendaal F, Wezel-Meijler GV. Neonatal care bundles are associated with a reduction in the incidence of intraventricular haemorrhage in preterm infants: a multicentre cohort study. Arch Dis Child Fetal Neonatal Ed. 2020;105(4):419-24.

17. Bass WT. Elevated midline head positioning of extremely low birth weight infants: effects on cardiopulmonary function and the incidence of periventricularintraventricular hemorrhage. J Perinatol. 2019;39(3):517-8.

18. Aarnivala H, Vuollo V, Harila V, Heikkinen T, Pirttiniemi P, Valkama AM. Preventing deformational plagiocephaly through parent guidance: a randomized, controlled trial. Eur J Pediatr. 2015;174(9):1197-208.

19. Kobesova A, Kolar P. Developmental kinesiology: three levels of motor control in the assessment and treatment of the motor system. J Bodyw Mov Ther. 2014; 18(1):23-33.

20. Ferreira CT, Carvalho E, Sdepanian VL, Morais MB, Vieira MC, Silva LR. Doença do refluxo gastroesofágico: exageros, evidência e a prática clínica. J Pediatr (Rio J). 2014;90(2):105-18.

Recebido:06 dez, 2020 Aceito: 29 mar, 2021 